\title{
Introduction to the special topic Embodied and Grounded Cognition
}

\author{
Anna M. Borghi ${ }^{1 * t}$ and Diane Pecher ${ }^{2 * t}$ \\ University of Bologna and National Research Council, Rome, Italy \\ 2 Psychology Department, Erasmus University Rotterdam, Rotterdam, Netherlands \\ *Correspondence: anna.borghi@gmail.com; pecher@fsw.eur.nl \\ ${ }^{+}$Anna M. Borghi and Diane Pecher have contributed equally to this work.
}

\section{INTRODUCTION}

In the last $10-15$ years, the embodied and grounded (E and $\mathrm{G})$ cognition approach has become widespread in all fields related to cognitive (neuro) science, and a lot of evidence has been collected. The approach proposes that cognitive activity is grounded in sensory-motor processes and situated in specific contexts and situations.

This special topic had two aims: first, give an idea of the field in its broadness. Second, focus on some challenges for $\mathrm{E}$ and $\mathrm{G}$ theories. The first important challenge is to account for understanding abstract concepts and words. Evidence on the representation of concrete concepts is compelling, whereas evidence on abstract concepts is still scarce and limited to restricted domains. A second important challenge concerns the role of computational models. E and G theories of cognition need to formulate more precise hypotheses, and models help to constrain and specify in more detail the predictions and the claims advanced.

\section{THE FIELD IN ITS BROADNESS}

Although the importance of sensory-motor grounding had already become apparent in philosophy and linguistics, only after a couple of influential theoretical papers in the late 1990s did cognitive psychology get involved seriously (Glenberg, 1997; Zwaan and Radvansky, 1998; Barsalou, 1999; Pulvermüller, 1999). The idea that cognitive processes, such as those involved in language and memory, are grounded in the same systems as those used for perception and action has received much empirical support. This special topic presents a sample of the new and exciting empirical work in this field.

Grounding of language comprehension has been among the most compelling demonstrations. Several papers further investigated the grounded or embodied nature of a variety of linguistic issues. Wellsby et al. (2010) showed that people recognize embodied insults faster than less embodied insults. Hald et al. (2011) and Collins et al. (2011) using ERPs, both showed that the modality switch effect reflects modality differences in early meaning activation due to different sensory systems involved in the mental simulation. In an fMRI study, Rueschemeyer et al. (2010) showed that when people process motion language they simulate seeing motion and preparing for the actions that the situation requires. Thus, mental simulations are immediately affected by the sentence context. A similar conclusion is drawn by van Dam et al. (2010) who showed that the immediate linguistic context modulates whether object names activate action simulations. In contrast, Hemeren and Thill (2011) found that visual perception of functional object use is little affected by knowledge of the object identity. Instead, participants used kinematic information such as velocity, acceleration, and changes in direction to segment the action. Marsh and Glenberg (2010) propose that people learn grammar by imitation, in particular neuromuscular tuning. Lynott and Connell (2010) propose a grounded model (embodied conceptual combination, ECCo) for new combinations of familiar concepts such as elephant complaint. They argue that people mesh motor and perceptual affordances either in a destructive or non-destructive manner.

How do we understand other people? The social Simon effect supports the idea that we understand other people's actions by representing them as our own. Dolk et al. (2011) and Vlainic et al. (2010) found that online information from another person is not necessary, suggesting that the effect is not due to co-representation of the other's action but rather to awareness of a second location that highlights the congruency between stimulus location and response. Kessler and Rutherford (2010) showed that whether representations of other people's perspective are embodied depends on difficulty. They argue that the degree of symmetry determines whether perspective taking is needed.

\section{THE CHALLENGES THE CHALLENGE TO ACCOUNT FOR ABSTRACT CONCEPTS}

Most studies of $\mathrm{E}$ and $\mathrm{G}$ cognition have focused on concrete objects and actions. People can also represent and reason about abstract concepts that do not have many sensory-motor features, however, and there is not much evidence yet on grounding of abstract concepts in sensory-motor systems (for a review, see Pecher et al., 2011). Thus, critics have argued that current evidence does not fully account for abstract representation.

Some authors propose a theoretical solution. van Elk et al. (2010) challenged the reliance on representations and proposed an enactivist approach. They argued that the view of representations as simulations or re-enactment of previous experiences opens two problems. First, the necessity of sensory-motor systems for cognition has been disputed (e.g., Mahon and Caramazza, 2008). Second, it fails to explain concepts beyond our motor repertoire, such as animal actions, or abstract words. The authors proposed that sensory-motor brain areas underlie prediction of actions, arguing in favor of a more procedural view of cognition. In contrast, Dove believed that "the notion of representation is too useful to give up." Dove proposed to use the term dis-embodiment. Language is dis-embodied because its sensory-motor features are unrelated to its meaning. According to Dove (2011), this dual functionality of language is at the basis of generalization and abstraction.

Several papers addressed conceptual metaphor theory. Flusberg et al. (2010) presented a computational connectionist model 
of grounding time in the representation of space. Kranjec and Chatterjee (2010) challenged the view that time is grounded in space and investigated grounding of temporal concepts directly in neural areas dedicated to time perception. Beside conceptual metaphor theory, new views emerge that argue for multiple representations and assign a specific role to language (e.g., Barsalou et al., 2008). Views differ, however, on the role of symbolic associations. Whereas Dove (2011) proposes that symbolic processing complements sensory-motor processing, others have argued that symbolic associations merely provide shortcuts for more efficient task performance (Barsalou et al., 2008). Pecher and Boot (2011) found that spatial attention was affected by number magnitude, but, contrary to what conceptual metaphor theory would predict, only for numbers in concrete contexts. Associative shortcuts might suffice for abstract magnitudes, whereas concrete magnitudes require deeper processing. Borghi et al. (2011) investigated how the kind of acquisition influenced representation of concrete and abstract novel words. Verbal information is more crucial to represent abstract concepts, manual information to represent concrete concepts. This is in line with the proposal that Words are social Tools (Borghi and Cimatti, 2009): labels and explanations are particularly helpful to learn abstract words, as they provide the glue helping us to keep together multifaceted experiences.

Barbey and Patterson (2011) addressed abstraction from a cognitive neuroscience perspective. They reviewed evidence that PFC extracts statistical regularities across experiences; these regularities are the basis for building abstract rules, such as causal beliefs. The underlying mechanism is given by a distributed neural network across both modality specific and associative areas. This mechanism can be activated also when stimuli are not present, thus causing a causal simulation of an event.

\section{THE CHALLENGE OF COMPUTATIONAL MODELS}

The need for computational models emerges clearly. Pezzulo et al. (2011) argued that embodied robotics is the platform to test embodied computational models independent from the kind of modeling style - dynamic models, Bayesian approaches, or connectionist models. Robots provide cognitive process models with constraints because their sensory-motor potential and limitations can be made similar to those of humans.

Instead of a system that has a full representation of the environment, Rothkopf and Ballard (2010) proposed a system of simple visuomotor modules, learned through reinforcement, allowing rapid access to behavioral primitives. The authors illustrate a credit assignment algorithm that solves the problem of calibrating different visuomotor modules while pursuing multiple goals. In contrast, Ursino et al. (2010) presented a two-store model of representation: a semantic store of topologically organized sensory-motor features and a lexicon. Because attractors are not steady states but rather synchronized oscillations multiple objects can be represented at the same time. A time dependent Hebbian learning rule allows the model to learn the relationship between words and object features.

Parisi (2011) argued that current theories are limited because they ignore emotions, "the other half of the embodied mind." He argued that robots endowed with emotional circuits reach higher level of performance and have better survival probability compared to robots without emotion. Mizelle and Wheaton (2010) criticized current theories for ignoring flexibility. Their modular MSAG model represents high plasticity and variability in tool selection and use.

\section{CONCLUSION}

We believe this special topic has opened new perspectives, and gives us some indications of where the field of $E$ and $G$ cognition is going. First of all, it testifies that the domain has expanded rapidly. At the same time, however, researchers will have to solve some open issues, such as the questions of necessity and abstraction. This special topic presents many empirical, theoretical, and modeling approaches to these challenges. It is a proof of how lively and open the field is.

\section{REFERENCES}

Barbey, A., and Patterson, R. (2011). Architecture of explanatory inference in the human prefrontal cortex. Front. Psychol. 2:162. doi: 10.3389/fpsyg.2011.00162 Barsalou, L. W. (1999). Perceptual symbol systems. Behav. Brain Sci. 22, 577-660.

Barsalou, L. W., Santos, A., Simmons, W. K., and Wilson, C. D. (2008). "Language and simulation in conceptual processing," in Symbols, Embodiment, and Meaning, eds M. De Vega, A. M. Glenberg, and A. C. Graesser (Oxford: Oxford University Press), 245-283.
Borghi, A. M., and Cimatti, F. (2009). "Words as tools and the problem of abstract words meanings," in Proceedings of the 31st Annual Conference of the Cognitive Science Society, eds N. Taatgen and H. van Rijn (Amsterdam: Cognitive Science Society), 2304-2309.

Borghi, A. M., Flumini, A., Cimatti, F., Marocco, D., and Scorolli, C. (2011). Manipulating objects and telling words: a study on concrete and abstract words acquisition. Front. Psychol. 2:15. doi: 10.3389/fpsyg.2011.00015

Collins, J., Pecher, D., Zeelenberg, R., and Coulson, S. (2011). Modality switching in a property verification task: an ERP study of what happens when candles flicker after high heels click. Front. Psychol. 2:10. doi: 10.3389/fpsyg.2011.00010

Dolk, T., Hommel, B., Colzato, L. S., Schutz-Bosbach, S., Prinz, W., and Liepelt, R. (2011). How 'social' is the social Simon effect? Front. Psychol. 2:84. doi: 10.3389/ fpsyg.2011.00084

Dove, G. (2011). On the need for embodied and disembodied cognition. Front. Psychol. 1:242. doi: 10.3389/fpsyg.2010.00242

Flusberg, S. J., Thibodeau, P. H., Sternberg, D. A., and Glick, J. J. (2010).A connectionist approach to embodied conceptual metaphor. Front. Psychol. 1:197. doi: 10.3389/fpsyg.2010.00197

Glenberg,A.M. (1997). What memory is for. Behav. Brain Sci. 20, 1-55.

Hald, L. A., Marshall, J., Janssen, D. P., and Alan, G. (2011). Switching modalities in a sentence verification task: ERP evidence for embodied language processing. Front. Psychol. 2:45. doi: 10.3389/fpsyg.2011.00045

Hemeren, P.E., and Thill, S. (2011). Deriving motor primitives through action segmentation. Front. Psychol. 1:243. doi: 10.3389/fpsyg.2010.00243

Kessler, K., and Rutherford, H. (2010). The two forms of visuo-spatial perspective taking are differently embodied and subserve different spatial prepositions. Front. Psychol. 1:213. doi: 10.3389/fpsyg.2010.00213

Kranjec, A., and Chatterjee, A. (2010). Are temporal concepts embodied? A challenge for cognitive neuroscience. Front. Psychol. 1:240. doi: 10.3389/ fpsyg.2010.00240

Lynott, D., and Connell, L. (2010). Embodied conceptual combination. Front. Psychol. 1:212. doi: 10.3389/ fpsyg.2010.00212

Mahon, B., and Caramazza, A. (2008). A critical look at the embodied cognition hypothesis and a new proposal for grounding conceptual content. J. Physiol. Paris 102, 59-70.

Marsh, E. R., and Glenberg, A. M. (2010). The embodied statistician. Front. Psychol. 1:184. doi: 10.3389/ fpsyg.2010.00184

Mizelle, J. C., and Wheaton, L. A. (2010). The neuroscience of storing and molding tool action concepts: How "plastic" is grounded cognition? Front. Psychol. 1:195. doi: 10.3389/fpsyg.2010.00195

Parisi, D. (2011). The other half of the embodied mind. Front. Psychol. 2:69. doi: 10.3389/fpsyg.2011.00069

Pecher, D., and Boot, I. (2011). Numbers in space: differences between concrete and abstract situations. Front. Psychol. 2:121. doi: 10.3389/fpsyg.2011.00121

Pecher, D., Boot, I., and van Dantzig, S. (2011). "Abstract concepts: sensory-motor grounding, metaphors, and beyond," in The Psychology of Learning and Motivation, Vol. 54, ed. B. Ross (Burlington: Academic Press), 217-248.

Pezzulo, G., Barsalou, L. W., Cangelosi, A., Fischer, M. H., Spivey, M., and McRae, K. (2011). The mechanics of embodiment: a dialogue on embodiment and com- 
putational modeling. Front. Psychol.2:5. doi: 10.3389/ fpsyg.2011.00005

Pulvermüller, F. (1999). Words in the brain's language. Behav. Brain Sci. 22, 253-336.

Rothkopf, C. A., and Ballard, D. H. (2010). Credit assignment in multiple goal embodied visuomotor behavior. Front. Psychol. 1:173. doi: 10.3389/ fpsyg. 2010.00173

Rueschemeyer, S., Glenberg, A. M., Kaschak, M. P., Mueller, K., and Friederici, A. D. (2010). Top-down and bottom-up contributions to understanding sentences describing objects in motion. Front. Psychol. 1:183. doi: 10.3389/fpsyg.2010.00183

Ursino, M., Cuppini, C., and Magosso, E. (2010). A computational model of the lexical-semantic system, based on a grounded cognition approach. Front. Psychol. 1:221. doi: 10.3389/fpsyg.2010.00221 van Dam, W. O., Rueschemeyer, S.-A., Lindemann, O., and Bekkering, H. (2010). Context effects in embodied lexical-semantic processing. Front. Psychol. 1:150. doi: 10.3389/fpsyg.2010.00150

van Elk, M., Slors, M., and Bekkering, H. (2010). Embodied language understanding requires an enactivist paradigm of cognition. Front. Psychol. 1:234. doi: 10.3389/fpsyg.2010.00234

Vlainic, E., Liepelt, R., Colzato, L. S., Prinz, W., and Hommel, B. (2010). The virtual co-actor: the social Simon effect does not rely on online feedback from the other. Front. Psychol. 1:208. doi: 10.3389/ fpsyg.2010.00208

Wellsby, M., Siakaluk, P. D., Pexman, P. M., and Owen, W. J. (2010). Some insults are easier to detect: the embodied insult detection effect. Front. Psychol. 1:198. doi: 10.3389/fpsyg.2010.00198
Zwaan, R. A., and Radvansky, G. A. (1998). Situation models in language comprehension and memory. Psychol. Bull. 123, 162-185.

Received: 29 June 2011; accepted: 22 July 2011; published online: 17 August 2011.

Citation: Borghi AM and Pecher D (2011) Introduction to the special topic Embodied and Grounded Cognition. Front. Psychology 2:187. doi: 10.3389/fpsyg.2011.00187 This article was submitted to Frontiers in Cognition, a specialty of Frontiers in Psychology.

Copyright $(2011$ Borghi and Pecher. This is an open-access article subject to a non-exclusive license between the authors and Frontiers Media SA, which permits use, distribution and reproduction in other forums, provided the original authors and source are credited and other Frontiers conditions are complied with. 